\title{
Hydrothermal waves in two-dimensional liquid layers with sudden changes in the available cross-section
}

\section{Marcello Lappa}

Dept. of Mechanical and Aerospace Engineering, University of Strathclyde 


\section{Hydrothermal waves in two-dimensional liquid layers with sudden changes in the available cross-section}

Purpose - Hydrothermal waves represent the preferred mode of instability of the so-called Marangoni flow for a wide range of liquids and conditions. The related features have attracted much attention over recent years owing to the relevance of these oscillatory modes to several techniques used for the production of single crystals of seminconductor or oxide materials. Control or a proper knowledge of convective instabilities in these systems is an essential topic from a material/product properties saving standpoint.

Design/methodology/approach - This short paper reports on a numerical model developed to inquire specifically about the role played by sudden changes in the available cross-section of the shallow cavity hosting the liquid. Although accounting for the spanwise dimension would be necessary to derive quantitative results, our approach is based on the assumption of twodimensional flow, which, for high-Pr fluids, is believed to retain the essence of the involved physical processes.

Findings - Results are presented for the case of a fluid with $\operatorname{Pr}=15$ filling an open container with a single backward-facing or forward-facing step on the bottom wall or with an obstruction located in the center. It is shown that the presence of steps in the considered geometry can lead to a variety of situations with significant changes in the local spectral content of the flow and even flow stabilization in certain circumstances. The role of thermal boundary conditions is assessed by considering separately adiabatic and conducting conditions for the bottom wall.

Originality/value - Although a plethora of studies have been appearing over recent years motivated, completely or in part, by a quest to discover means of mitigating instabilities and hence of producing single crystals of higher quality for the industry, unfortunately, however, most of these studies were focusing on very simple geometries. In the present paper, the causality and interdependence among all the kinematic and thermal effects mentioned above is discussed.

Keywords - Marangoni flow, hydrothermal waves, Finite difference method, variable section geometries.

\section{Introduction}

The rapid development that has taken place over the past thirty years in the field of electronic and optoelectronic devices has been fostered on the one hand by the increasing basic knowledge of solid-state physics and on the other hand by the amount of theoretical knowledge about the behavior of fluids. As a result of this new and sophisticated material engineering, methods have opened up the way for the production of compounds with characteristics particularly suitable for new applications. Among such techniques, e.g., it is worth citing the horizontal Bridgman (HB), the Floating zone (FZ) and the Czochralski (CZ) methods (see, e.g., Lappa, 2005).

Among the most promising materials, the socalled "transparent oxides" should be mentioned in particular. This category includes, e.g., $\mathrm{MgAl}_{2} \mathrm{O}_{4}$ (spinel), $\mathrm{Al}_{2} \mathrm{O}_{3}$ (sapphire), $\mathrm{Y}_{3} \mathrm{Al}_{5} \mathrm{O}_{12}$ (yttrium 
aluminium), $\mathrm{YAlO}_{3}$ (yttrium orthoaluminate), $\mathrm{Gd}_{3} \mathrm{Ga}_{5} \mathrm{O}_{12}$ (gadolinium gallium garnet), $\mathrm{LiTaO}_{3}$ (lithium tantalate).

These compounds are typically used for a variety of applications: from solid state lasers, magnetic bubble device substrates, insulating substrates for semiconductors, and monolithic crystal filters.

Another category of similar (transparent) oxides is represented by the so-called transparent conductive oxides (TCOs). Transparent conducting oxides such as Sn-doped $\operatorname{~}_{2} \mathrm{O}_{3}$ (indium tin oxide, ITO), Al-doped $\mathrm{ZnO}$, and $\mathrm{Sb}$-doped $\mathrm{SnO}_{2}$ are typically employed as transparent electrodes for liquid crystal displays (LCDs), organic light-emitting diodes (OLEDs), and solar cells. Moreover, such compounds are applicable to transparent optoelectronics because they have the unique features of optical transparency in the visible region and controllable electrical conductivity, from almost insulating to degenerate semiconducting behaviour (Ohta and Hosono, 2004).

A feature common to all processes for the production of such materials is the presence of thermal or compositional gradients, which are, in general, destabilizing, and provide driving forces for free convection in all fluid phases involved. These melt growth processes are, therefore, subject to varying heat- and mass-transfer conditions, which often result in undesired flows and ensuing instabilities.

When convection in the melt is oscillatory, in particular, the associated oscillations in the heat flux from the melt to the crystal produce fluctuations in the local growth rate, with alternating periods of growth and remelting. These effects are highly undesired and affect the performances of these materials in a very detrimental way. Control or a proper knowledge of convective instabilities in these systems is, therefore, an essential topic from a material/product properties saving standpoint.

A plethora of studies have been appearing over recent years motivated, completely or in part, by a quest to discover means of mitigating instabilities and hence of producing single crystals of higher quality for the industry. Unfortunately, however, most of these studies were focusing on idealized very simple geometries with weak relevance to effective single-crystalline materials production techniques. A large cross section of fundamental research on these topics has been reviewed, e.g., by Lappa (2004, 2007ab, 2009, 2012).

Along these lines, let us recall that in the presence of a free liquid/gas interface, the socalled Marangoni (thermocapillary) flow often plays a dominant role and, therefore, the aforementioned undesired effects must be ascribed primarily to the typical instability modes of this kind of convection. In many circumstances these modes correspond to the socalled hydrothermal waves originally predicted by Smith and Davis (1983) for the idealized case of a layer of infinite extent and observed by many other authors in more complex geometrical configurations (see, e.g., Bucchignani, 2004; Shevtsova et al., 2001, 2003, 2009, 2011; Lappa et al., 2000, 2003; Ueno et al., 2003 and Melnikov et al., 2004 and 2005; Lappa, 2013, 2014 and 2016; Schwabe et al., 1992 and 2003; Li et al., 2003 and 2006; Shi and Imaishi, 2006).

Such waves, which can be regarded as a classical example of flow established via a Hopf bifurcation (see, e.g., Chen et al., 2000), are oblique over the whole range of Prandtl number, i.e. they are threedimensional disturbances (in other words, they exhibit an angle of propagation relative 
to the basic state). The related angle of propagation, however, does depend on Pr: it is nearly perpendicular to the basic state for low-Pr materials, i.e. the disturbance propagates almost exactly in the spanwise direction (the wave has a longitudinal wavefront in such a case and for this reason it is generally referred to as longitudinal wave or disturbance); and nearly parallel to the surface flow for high-Pr materials such as oxide substances, i.e. the disturbance propagates almost exactly in the upstream direction (the waveforn in such a case being transverse).

Important extensions to the original work of Smith and Davis (1983) and the later weakly nonlinear analysis by Smith (1986) were provided by Priede and Gerbeth (1997), who analyzed the effect of various thermal boundary conditions on the linear stability of the parallel-flow solution. In particular, they addressed the more realistic case in which the adiabatic bottom is replaced by a conducting boundary (to mimick an external metallic container) with the free liquid/gas surface retaining an adiabatic behavior. In such a context it is also worth citing Shevtsova et al. (2014), who concentrated expressly on the dynamic coupling between the liquid and the external (gas) environment.

Despite these efforts, however, no attempts have been made to assess the role played by more realistic boundary conditions such as the presence of irregularities in the shape of the solid wall (in the form of bumps, steps, obstructions, etc.)

While for the companion case of buoyancy (thermogravitational) convection, some important studies have appeared for the specific case of flow over vertical, horizontal and inclined backwardand forward-facing steps (Abu-Mulaweh, 2002, 2003, 2005; Rouijaa et al., 2011; Meskini et al., 2011; Dihmani et al., 2012; Mahrouche et al., 2013), there seems to be a disappointing lack of equivalent knowledge for Marangoni flow.

In such a context, our article builds on, but also seeks to extend, the valuable research that over recent years has tried to enrich our fundamental understanding about the influence that kinematic and thermal (boundary) effects of various types can exert on the properties of the emerging Marangoni flow. More specifically, we consider the interplay of hydrothermal waves with sudden variations in the cross-sectional area of the considered cavity for high-Pr liquids (representative of the aforementioned transparent oxide materials), a case hitherto not addressed in the literature. Numerical examples are espressly conceived and presented to support the proper discernment of the cause-and-effect relationships underpinning the emergence of specific flow features in terms of waveforms and spectral content of the flow.

\section{Mathematical Model and Numerical Method}

\subsection{The liquid, the geometry and the assumption of two-dimensional flow}

The considered value of the Prandtl number $(\operatorname{Pr}=v / \alpha$ where $v$ is the fluid kinematic viscosity and $\alpha$ is the thermal diffusivity) is $\operatorname{Pr}=15$ ( 1 cs silicone oil). Many experiments are available in the literature where this or similar values of the Prandtl number were examined. Riley and Neitzel 
(1998) were the first to provide experimental evidence of oblique waves in a rectangular pool for silicone oil $(\mathrm{Pr}=14)$ for sufficiently thin layers in normal gravity conditions. Most of the available results for transparent liquids with $\mathrm{Pr}=\mathrm{O}(10)$ and normal gravity conditions (Daviaud and Vince, 1993 for $\operatorname{Pr}=10.3$; Gillon and Homsy, 1996 for $\operatorname{Pr}=9.5$; De Saedeleer et al., 1996 for $\operatorname{Pr}=15$; Garcimartìn et al., 1997 for $\operatorname{Pr}=10,15$ and 30; Pelacho and Burguete, 1999 and Pelacho et al., 2000 for $\operatorname{Pr}=10.3$ ) can be found (together with the related experimental setups, i.e. length, width and height of the containers used) in the analysis of Burguete et al. (2001), where it was highlighted how the emergence of a pattern with specific features depends essentially on geometrical factors. In such a context, it is also worth mentioning Pelacho et al. (2001), who performed a series of experiments expressly conceived to characterize the emergence of waves in rectangular containers whose dimensions (height, width and length) could be continuously changed; in this way, these authors were able to investigate how boundaries affect the threshold for the instability, as well as their consequences on other properties of the waves.

From all these results it is known that the angle formed by travelling waves with the direction of the surface flow is approximately $20^{\circ}$ for a value of the Prandtl number close to 15 .

Though proper capturing the spanwise component of these waves would require fully threedimensional (3D) simulations, following some other similar attempts in the literature (see, e.g., Peltier and Biringen, 1993; Xu and Zebib, 1998; Tang and $\mathrm{Wu}, 2005)$ in this analysis we make expressly the choice to collapse the $3 \mathrm{D}$ primitive equations to a two-dimensional (2D) one-layer model. Clearly, such reduction allows investigation of the considered subject in an "idealized" setting where excluded are any processes that depend on the details of the third (spanwise) dimension. Although accounting for the third dimension would be necessary to derive quantitative results, our approach stems from the two-fold intention to 1) look directly at the physical processes involved for high-Pr fluids, which are believed to be "retained" in the framework of a 2D model, 2) filter-out possible "localized" boundary effects produced by the presence of walls perpendicular to the third dimension and 3) reduce the otherwise prohibitive computational time that would be required by parametric $3 \mathrm{D}$ investigations.

For concreteness, we consider a shallow cavity with free liquid/gas interface parallel to the $\mathrm{x}$ axis and characteristic depth d, laterally delimited by solid walls at different temperatures (one cooled, the other heated). As a new feature with respect to earlier studies, our geometry (see Fig. 1) also incorporates a backward-facing (forward-facing) step or an obstruction located in the center (given by the combination of a backward-facing and forward-facing step).

The overall system aspect ratio (A), defined as its length-to-depth ratio (L/d) is fixed here to 20 . Other relevant characteristic geometrical parameters (refer to Fig. 1) are defined as $\Gamma=\mathrm{L}_{\mathrm{s}} / \mathrm{L}, \Gamma_{1}=$ $\mathrm{L}_{1} / \mathrm{L}$ and the expansion (compression) ratio $\Lambda=\mathrm{d}_{\mathrm{s}} / \mathrm{d}$, which clearly indicate that the simple act of introducing an obstacle opens up a vast parameter space that has not been explored yet, neither experimentally, nor numerically. 

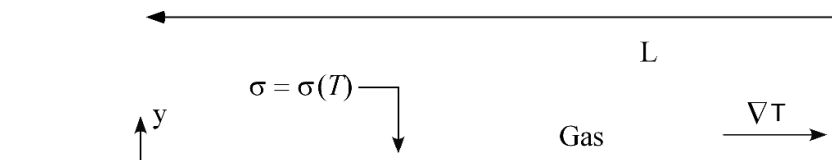

$\mathrm{T}_{\text {Cold }}$

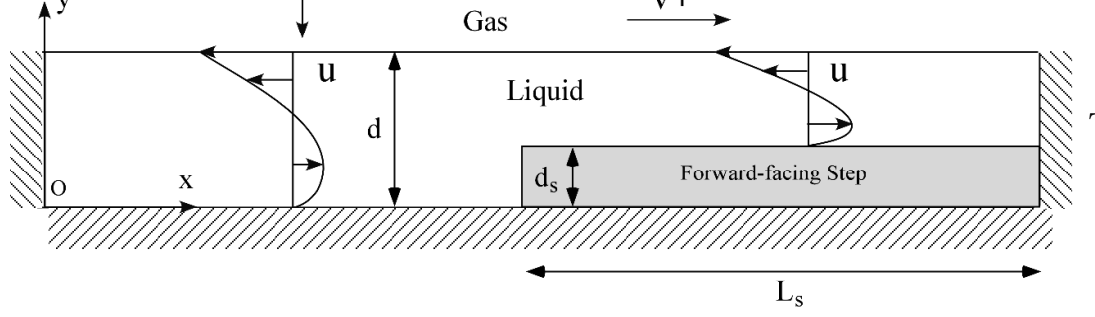

$\mathrm{T}_{\mathrm{Hot}}$

a)
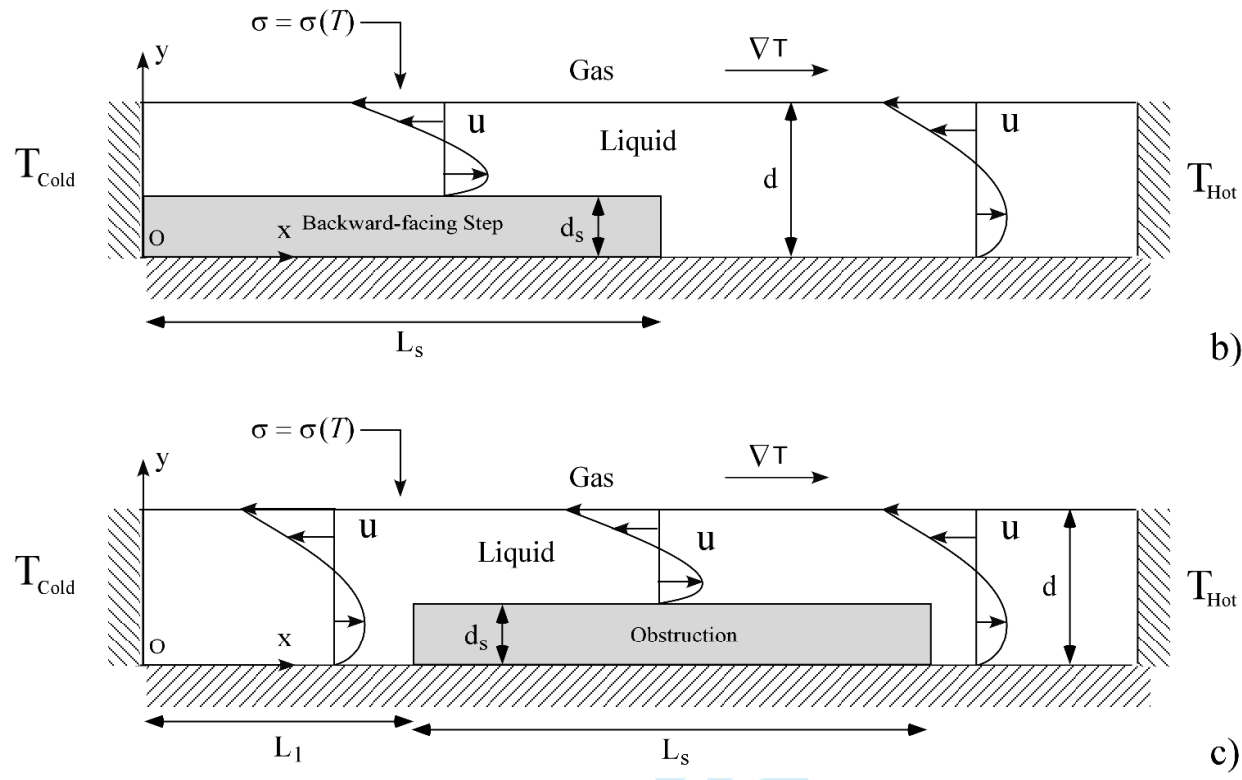

Figure 1: Sketch of the open cavity supporting the emergence and propagation of hydrothermal waves.

Such a system might be regarded as an ideal target to study the dynamics of hydrothermal waves in the presence of sudden changes in the geometry of the fluid container. Moreover, given the sensitivity of these waves to the thermal boundary conditions at the bottom wall shown by earlier studies (Priede and Gerbeth, 1997), both adiabatic and conducting behaviours are considered here (while the free liquid/gas interface is assumed to retain an adiabatic behaviour in all cases).

\subsection{The balance equations}

Using the conventional reference quantities, i.e. $d$ for the co-ordinates, $V_{\alpha}=\alpha / d, d^{2} / \alpha$ and $\rho \alpha^{2} / d^{2}$ (where $\rho$ is the fluid density), for velocity, time and pressure, respectively, the nondimensional momentum equation can be written as:

$\frac{\partial \underline{V}}{\partial t}=-\underline{\nabla p}-\underline{\nabla} \cdot[\underline{V} \underline{V}]+\operatorname{Pr} \nabla^{2} \underline{V}+\operatorname{Pr} R a T \underline{i}_{g}$ 
where $R a$ is the well-known Rayleigh number defined as $R a=\mathrm{g} \beta_{\mathrm{T}} \Delta \mathrm{Td}^{3} / v \alpha$ ( $\beta_{\mathrm{T}}$ being the thermal expansion coefficient and $\Delta \mathrm{T}$ the imposed temperature difference between the hot and cold sidewalls).

Accordingly, the continuity and energy equations can be cast in compact form as:

$\underline{\nabla} \cdot \underline{V}=0$

and

$\frac{\partial T}{\partial t}=-\underline{\nabla} \cdot[V T]+\nabla^{2} T$

where the nondimernsional temperature has been defined as $T=\left(\bar{T}-\bar{T}_{\text {cold }}\right) / \Delta \mathrm{T}$ ( $\bar{T}$ being the dimensional temperature). In the following, microgravity conditions are considered, i.e. Ra is set equal to zero.

\section{$\underline{2.3 \text { The Initial and Boundary conditions }}$}

The initial conditions are:

$\mathrm{t}=0: \quad \underline{\mathrm{V}}(\mathrm{x}, \mathrm{y})=0, \mathrm{~T}(\mathrm{x}, \mathrm{y})=\mathrm{x} / \mathrm{A}$

i.e. the liquid is motionless with a linear temperature profile along the $\mathrm{x}$ coordinate (the temperature is $\mathrm{T}_{\text {Cold }}=0$ on the cold sidewall and $\mathrm{T}_{\mathrm{Hot}}=1$ on the other side).

The boundary conditions at the free surface read:

$\mathrm{v}=0, \partial \mathrm{u} / \partial \mathrm{y}=-\mathrm{Ma} \partial \mathrm{T} / \partial \mathrm{x}$ and $\partial \mathrm{T} / \partial \mathrm{y}=0$ at $\mathrm{y}=1$ (free surface)

where $u$ and $v$ are the velocity components along $\mathrm{x}$ and $\mathrm{y}$, respectively, and

$M a=\sigma_{\mathrm{T}} \Delta \mathrm{Td} / \mu \alpha$

( $\mu$ being the dynamic viscosity) is the socalled Marangoni number. This condition enforces a flow by tangential variation of the surface tension.

Moreover, no slip conditions $(u=v=0)$ are set on all the solid walls.

The temperature is assumed to be constant at the right and left sidewalls $\left(\mathrm{T}_{\text {Cold }}=0, \mathrm{~T}_{\text {Hot }}=1\right)$, while it satisfies the following conditions on the lower solid boundary: 
$\partial \mathrm{T} / \partial \mathrm{n}=0$

(where $\mathrm{n}$ is the direction locally perpendicular to the bottom boundary, namely, $\mathrm{y}$ if the considered portion of boundary is horizontal or $\mathrm{x}$ if it is vertical) or

$T(x, y)=x / A$

according to whether the wall is assumed to be adiabatic or conducting (in the latter case, as specified by eq. (8), the distribution of temperature is linear if the considered portion of the bottom boundary is horizontal and it becomes constant if such a portion has a vertical orientation).

\subsection{The Numerical method}

Balance equations and related boundary conditions (1-8) have been solved in their complete nonlinear and time-dependent form using a projection method, that is an approach by which the computation of the velocity field is articulated into two main macro steps providing separately an intermediate field that has the correct content of vorticity but does not satisfy the continuity equation and a final velocity field, which has both the correct values of divergence and curl. Such a field is "unique" according to the Ladyzhenskaya decomposition theorem (Ladyzhenskaya, 1969), which states that any vector function can be split into a part of given divergence and the gradient of a scalar potential. Related details can be found for instance in Harlow and Welch (1965), Chorin (1968) and Temam (1968). These techniques have been very studied since those works (relevant and important extensions were provided, e.g., by Gresho, 1991; Quartapelle, 1993; Guermond and Quartapelle, 1998; Lappa and Savino, 1999; Guermond et al., 2006, etc). Despite some minor differences, basically, a common ingredient of all these variants is their "turning around" the coupling between the pressure and the velocity that is established for incompressible fluids. These techniques are known under several other names such as: fractional-step method or pressurecorrection method (also simply referred to as primitive-variables approach). Our approach proceeds as a type of fractional step method by first considering a simplified momentum equation where pressure is neglected:

$$
\underline{V}^{*}=\underline{V}^{n}+\Delta t\left[-\underline{\nabla} \cdot(\underline{V} \underline{V})+\operatorname{Pr} \nabla^{2} \underline{V}\right]^{n}
$$

(where the $\mathrm{n}$ superscript denotes the time step), then solving the elliptic equation resulting from the substitution of the velocity field $\underline{V}^{\mathrm{n}+1}$ into the continuity equation:

$$
\nabla^{2} p^{n}=\frac{1}{\Delta t} \underline{\nabla} \cdot \underline{V}^{*}
$$


and finally updating the intermediate velocity field using the computed pressure to account for the conservation of mass:

$\underline{V}^{n+1}=\underline{V}^{*}-\Delta t \underline{\nabla} p^{n}$

By using the effective (physical) boundary conditions for eq. (9), the intermediate velocity field needs not to be corrected on the boundaries, which leads to the possibility to impose $\underline{\nabla p}=0$ there. Gresho and Sani (1987), in particular, were the first to demonstrate that the most convenient boundary condition (in terms of problem well-posedness) is simply the homogeneous Neumann condition that results from projecting the pressure gradient in a direction perpendicular to the boundary, namely $\partial \mathrm{p} / \partial \mathrm{n}=0$.

Here such an approach has been implemented on a rectangular mesh with a staggered collocation of fluid-dynamic variables, as usual with this class of techniques. In particular, forward differences in time and central-differencing schemes in space (second order accurate) have been used to discretize the energy and momentum governing equations.

Along these lines, it is also worth providing some useful information about the grid refinement study and the strategy that we had to implement to guarantee a proper resolution of all spatial and temporal scales involved in the considered phenomena.

\begin{tabular}{rl}
\hline Grid $\mathrm{N}_{\mathrm{x}} \times \mathrm{N}_{\mathrm{y}}$ & $\Omega_{\mathrm{HTW}}$ \\
\hline \hline $200 \times 20$ & 49.5 \\
$400 \times 20$ & 47.3 \\
$300 \times 30$ & 46.2 \\
$400 \times 30$ & 45.4 \\
$600 \times 30$ & 45.3 \\
\hline \hline
\end{tabular}

Table I: Grid Refinement Study: Angular frequency of the Hydrothermal Wave as a function of mesh resolution $\left(\mathrm{Pr}=15, \mathrm{~A}=20, \mathrm{Ma}=2 \times 10^{4}\right)$.

As representative conditions for the spatial grid refinement study, we have considered $\mathrm{Ma}=2 \times 10^{4}$ for which the solution is expected to be affected by the presence of lateral thermal boundary layers (whose proper resolution is essential to ensure grid independency). As sensitive parameters to check grid independency, we have considered the angular frequency of the hydrothermal wave $\left(\Omega_{\mathrm{HTW}}\right)$. The minimum mesh required to guarantee independence of such a frequency from the used spatial resolution has been found to be 30 points per unit nondimensional length. Accordingly a mesh with 600 points in the $\mathrm{x}$ direction and 60 points in the $\mathrm{y}$ direction has been used for all simulations (the number of points in the vertical direction has been doubled with respect to the minimum required to attain mesh independence to ensure proper resolution when the vertical depth of the layer is locally reduced due to the presence of obstacles). The resulting nondimensional spatial steps are $\Delta \mathrm{x} \cong 3.3 \times 10^{-2}$, and $\Delta \mathrm{y} \cong 1.7 \times 10^{-2}$, respectively. The corresponding time integrations step, resulting 
from the application of typical analytic criteria to guarantee algoritm numerical stability, is $\Delta \mathrm{t} \cong$ $2 \times 10^{-6}$.

\subsection{Code validation}

The code has been validated through comparison with the numerical results of Xu and Zebib (1998) for pure Marangoni flow in a rectangular cavity with $\mathrm{A}=20, \mathrm{Pr}=10$ and $\mathrm{Ma}=1.05 \times 10^{4}$. Using the same grid density that, according to the refinement study carried out in Sect. 2.4, guarantees a mesh-independent solution (for even larger values of the Prandtl and Marangoni number), we have obtained a traveling-wave angular frequency $\omega \cong 36$ matching with a reasonable approximation $(\cong 2 \%)$ the value $(35.17)$ reported by these authors.

\section{Results}

The numerical simulations have been carried out assuming the following conditions: $A=20$, $\mathrm{Ma}=2 \times 10^{4}, \Gamma=0.5, \Gamma_{1}=0.25$ (where applicable) and $0.25 \leq \Lambda \leq 0.45$. As anticipated in the introduction, we would like to draw attention to a few features that do not seem to have received much attention in past studies on these subjects. Motivated by this observational tide, first we survey some basic dynamical principles which seem to be applicable to all the results provided by the numerical simulations, then results of specific calculations are discussed in detail (to highlight hidden or overlooked aspects). Most conveniently, we start from the classical case with no step or obstructions (canonical rectangular open cavity) to illustrate the general property of the hydrothermal wave (HTW), then the discussion progresses via comparison with the cases affected by sudden changes in the geometry external boundary.

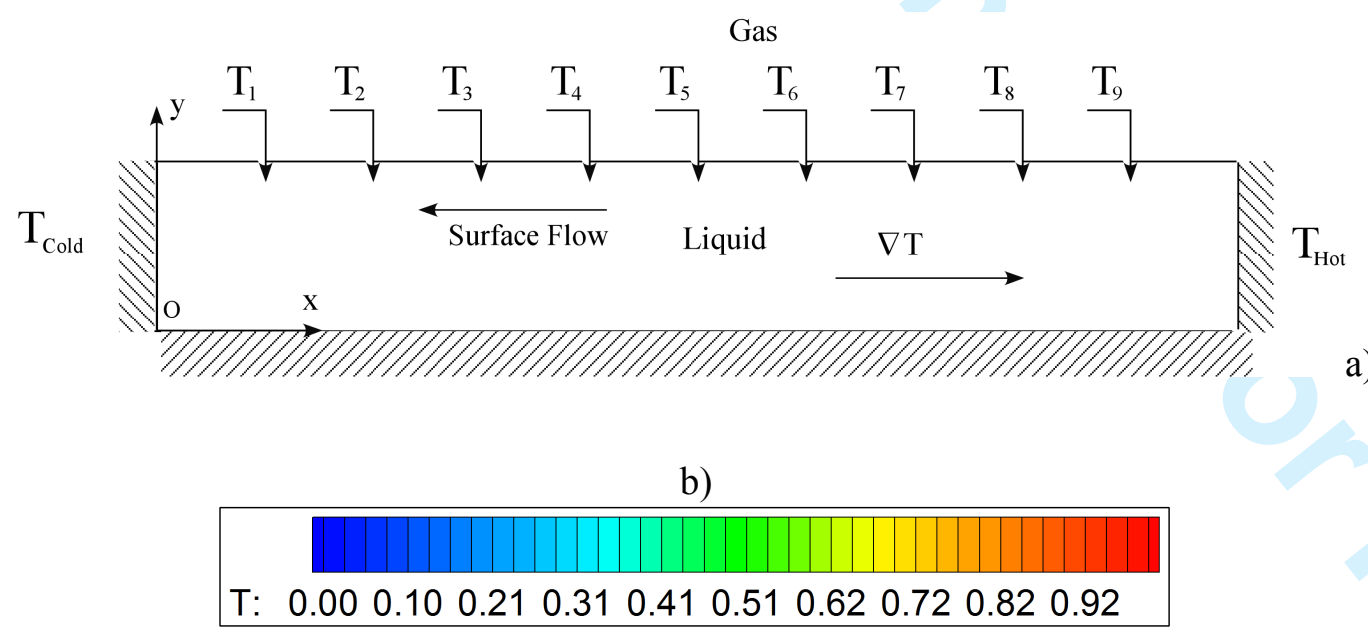

Figure 2: (a) Distribution of numerical probes along the cavity; (b) contour legend related to temperature maps. 


\subsection{The classical rectangular open cavity}

The general properties of the the hydrothermal waves in layers of infinite extent or geometries of finite size are well known since the works by Smith and Davis (1983), Peltier and Biringen (1993), Priede and Gerbeth (1997), Xu and Zebib (1998) and Bucchignani (2004). In the present paper, for illustration purposes, the behaviour of the temperature field as a function of time is expressly monitored at nine positions along the free liquid/gas interface. More specifically, nine "numerical probes", evenly spaced along the horizontal extension of the cavity as shown in Fig. 2, are used to obtain meaningful information of the propagation of the thermal disturbance. Temperature contour maps are provided as well (the reader is referred to Fig. $2 b$ for the related legend).
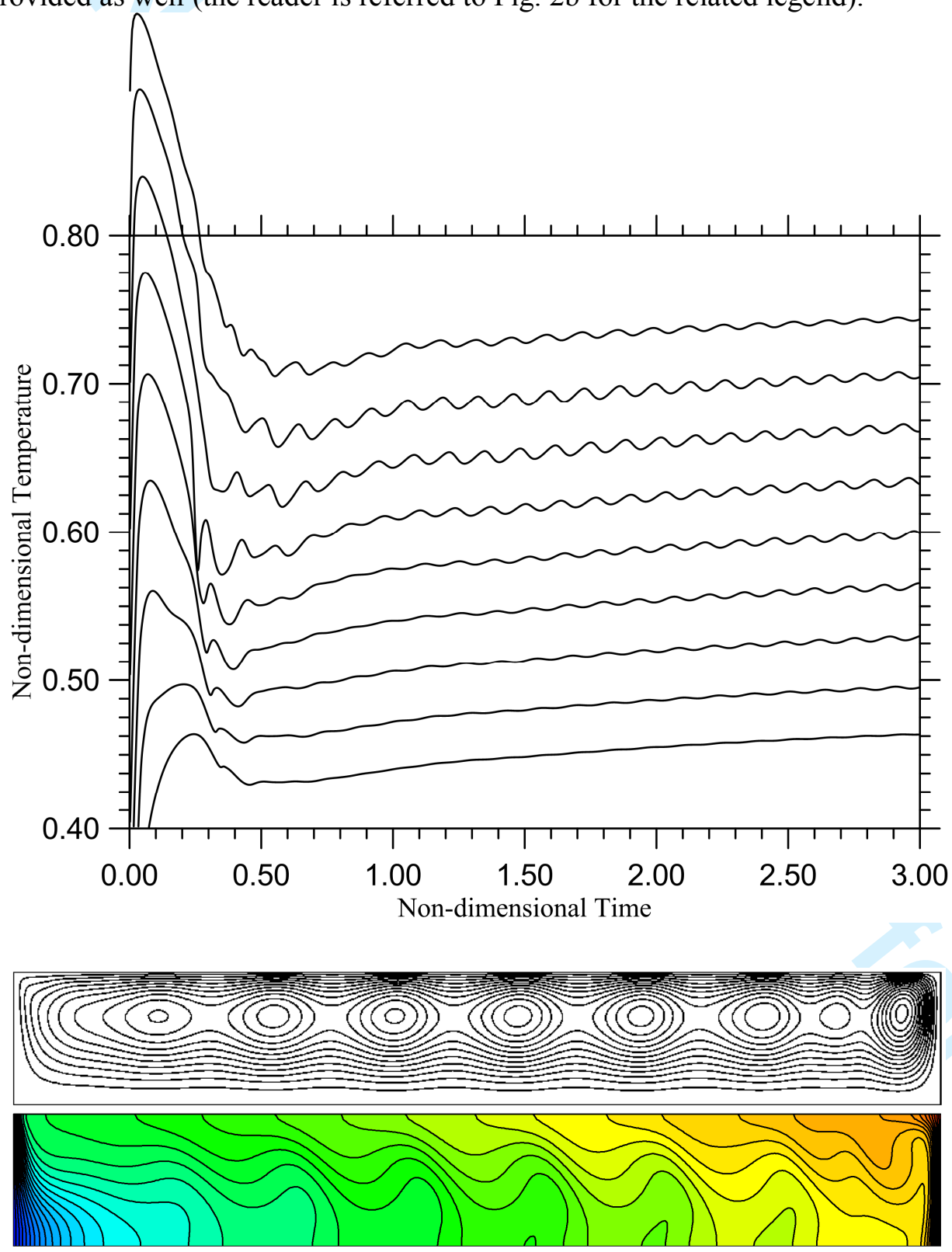

Figure 3: No step, adiabatic bottom: a) plot of signals provided by numerical probes, b) snapshot of streamlines $\left(\psi_{\min }=1.3, \psi_{\max }=17.63\right)$ and $\left.\mathrm{c}\right)$ temperature field at $\mathrm{t}=10$. 

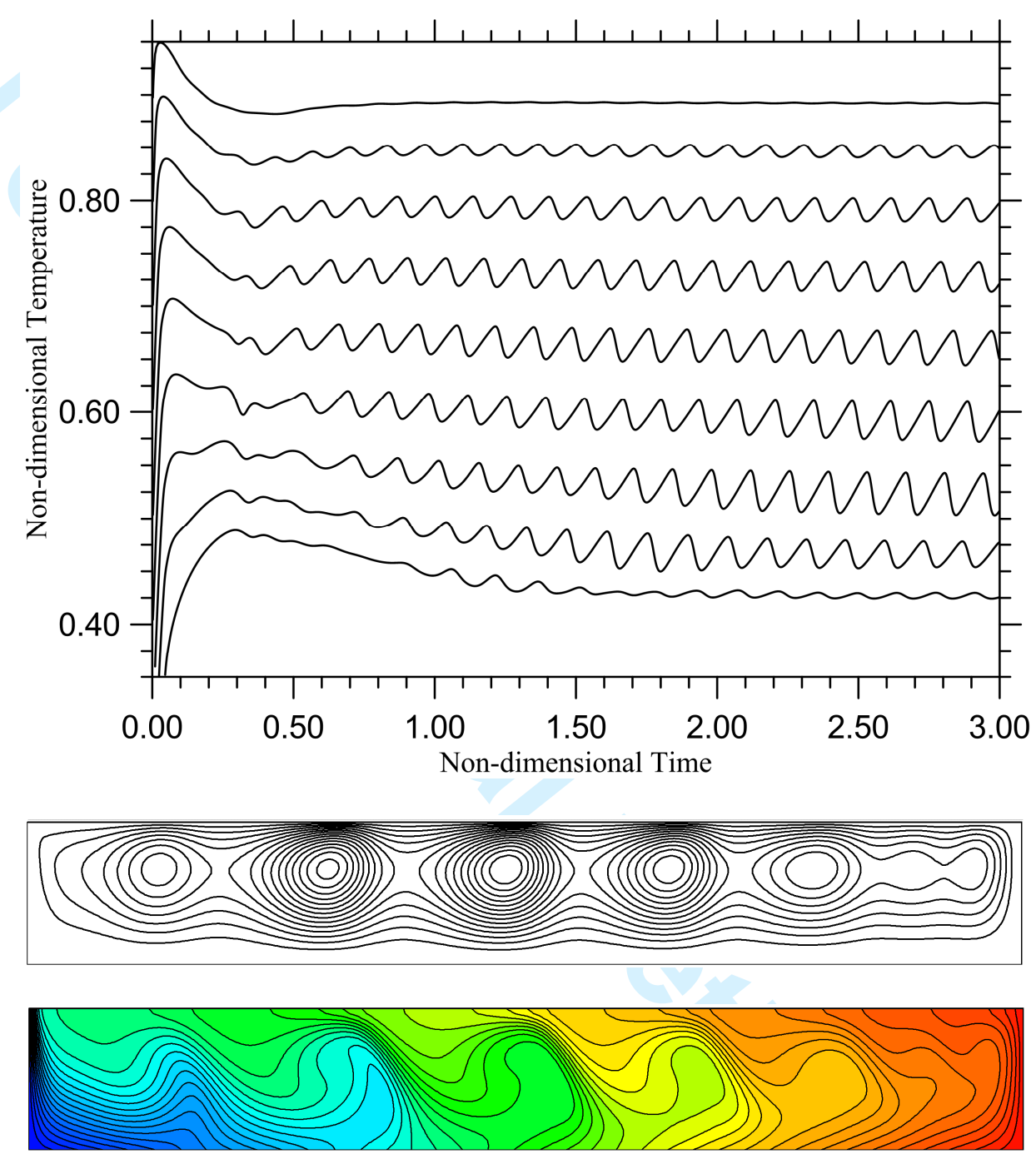

Figure 4: No step, conducting bottom: a) plot of signals provided by numerical probes, b) snapshot of streamlines $\left(\psi_{\min }=2.5, \psi_{\max }=35.2\right)$ and $\left.\mathrm{c}\right)$ temperature field at $\mathrm{t}=3$.

The numerical results for both adiabatic and conducting bottom wall are shown in Figs. 3 and 4, respectively. Apart from some expected differences, in terms of frequency and amplitude of the oscillations, due to the different behaviour of the temperature field in proximity to the bottom wall, some common characteristics can be highlighted. Indeed, in agreement, with earlier studies, it can be seen that the main disturbance consists essentially of a perturbation traveling in the upstream direction. As a result of such a disturbance, in particular, a series of rolls embedded in the overall circulation system (with fluid traveling from the hot side to the cold side at the free surface and in the opposite direction close to the bottom) become a persistent feature of the velocity field. These rolls are not stationary. Rather, by spreading continuously from the cold side to the hot side, they 
create the illusion of an endless traveling train of vortices. An almost steady vortex is manintained only in proximity to the hot wall where the temperature gradients established in the side thermal boundary layer are relatively strong.

The migration of rolls from the cooled wall to the heated one results in clear oscillations of the temperature measured at different locations along the horizontal coordinate. The amplitude of these oscillations is more or less constant along the horizontal extension. The related frequency is an intrinsic property of the oscillatory field, i.e. it is constant along $\mathrm{x}$.

\section{$\underline{3.2 \text { Forward-facing step }}$}

Direct comparison of the numerical results for this case with the canonical open cavity (no steps on the bottom) gives immediate insights into the effect of sudden changes in the geometry on the properties of the resulting HTW. Indeed, the reader will easily realize that the constancy of frequency and amplitude of temperature signal is no longer a feature of the field (amplitude and frequency of the traveling disturbance change significantly along the horizontal coordinate with respect to the case with straight bottom).

In particular, for $\Lambda=0.25$ the amplitude of oscillations decreases in the region with reduced crosssectional area $(x>A / 2)$ whereas their frequency undergoes an increase (Figs. 5 and 6$)$.

Such changes are reflected in observable modifications of the flow structure and related spatiotemporal evolution. The size of the rolls is much larger before the step. Furtermore their velocity of propagation undergoes a strong increase after the step.

Interestingly, although the changes experienced by the thermofluid-dynamic field are qualitatively similar for both adiabatic and conducting bottom walls, some differences can be highligted.

On average the number of rolls is $\mathrm{N}=9$ and $\mathrm{N}=7$ for adiabatic and conducting wall, respectively. What is even more remarkable, in the latter case the amplitude of temperature oscillations in the pre-step region $(\mathrm{x}<\mathrm{A} / 2)$ is much larger, while complete stabilization of the flow can be observed in proximity to the hot wall (see the top signal in Fig. 6, it displays no oscillations). 

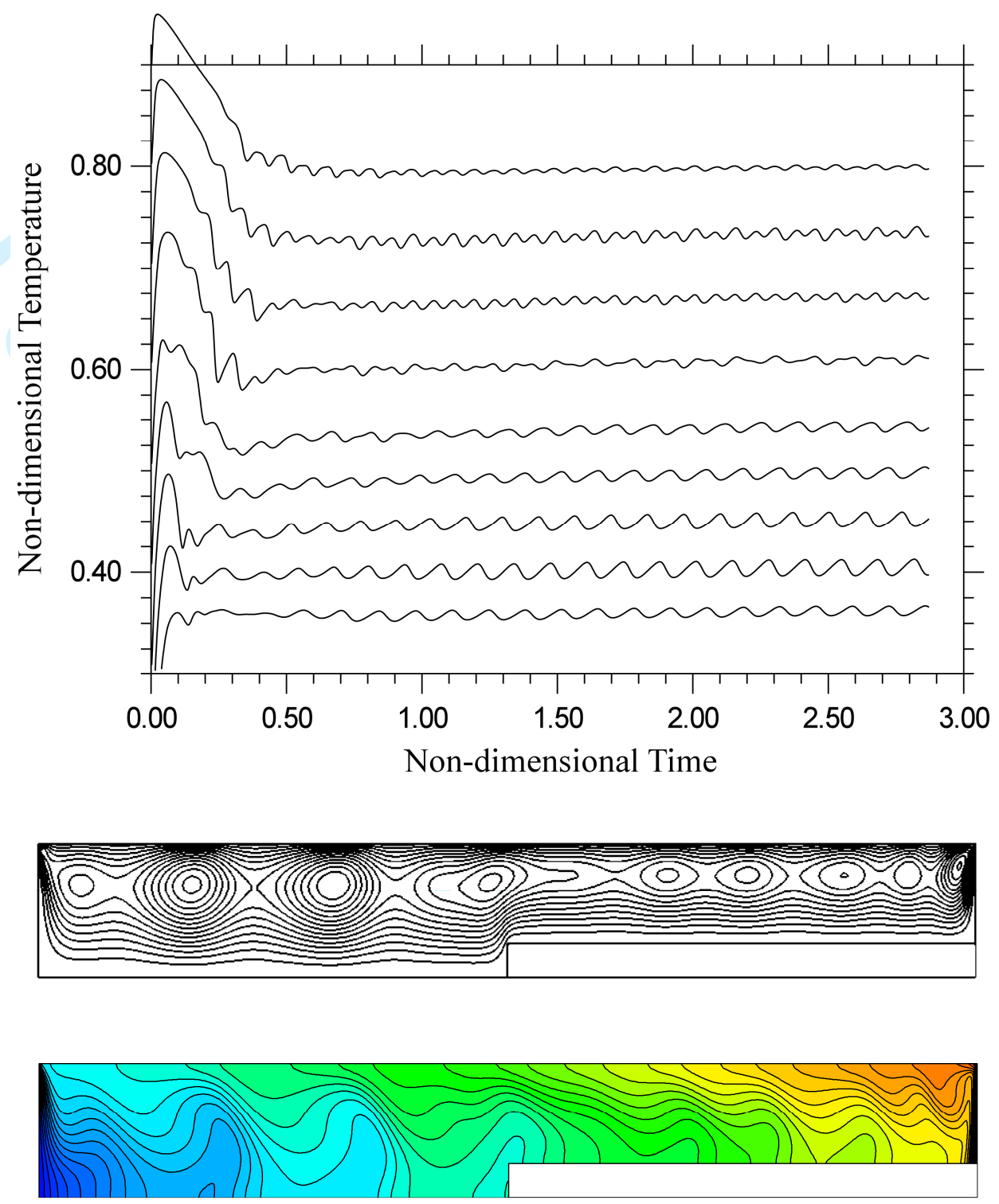

Figure 5: Forward-facing step, $\Lambda=0.25$, adiabatic bottom: a) plot of signals provided by numerical probes, $\mathrm{b})$ snapshot of streamlines $\left(\psi_{\min }=1.8, \psi_{\max }=24.9\right)$ and $\left.\mathrm{c}\right)$ temperature field at $\mathrm{t}=3$. 

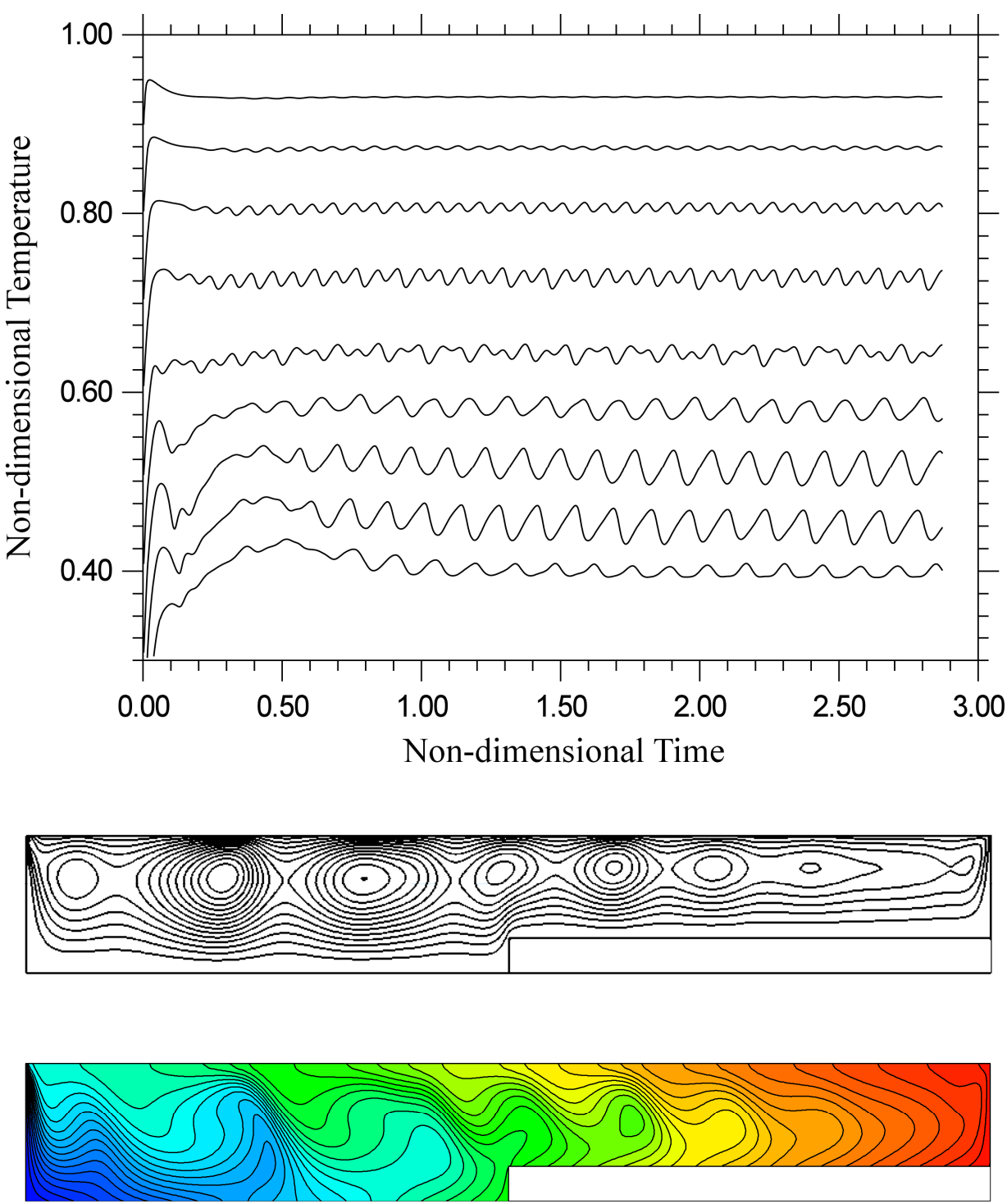

Figure 6: Forward-facing step, $\Lambda=0.25$, conducting bottom: a) plot of signals provided by numerical probes, b) snapshot of streamlines $\left(\psi_{\min }=2.7, \psi_{\max }=37.3\right)$ and c) temperature field at $\mathrm{t}=3$.

Notably, an increase in $\Lambda(\Lambda=0.45)$ leads to an almost complete stabilization of the flow in the step region $(x>A / 2)$ for both cases with an ensuing decrease of the average number of rolls to $N \cong 4$ (the HTW is limited to the left part of the cavity). For the adiabatic bottom case conditions of an almost parallel flow are attained in the post step region $(x>A / 2$, Fig. 7). However, this is not achieved for the other case (not shown) for which, among other things, like the case with $\Lambda=0.25$, the temperature oscillations are stronger in the region affected by the HTW. 

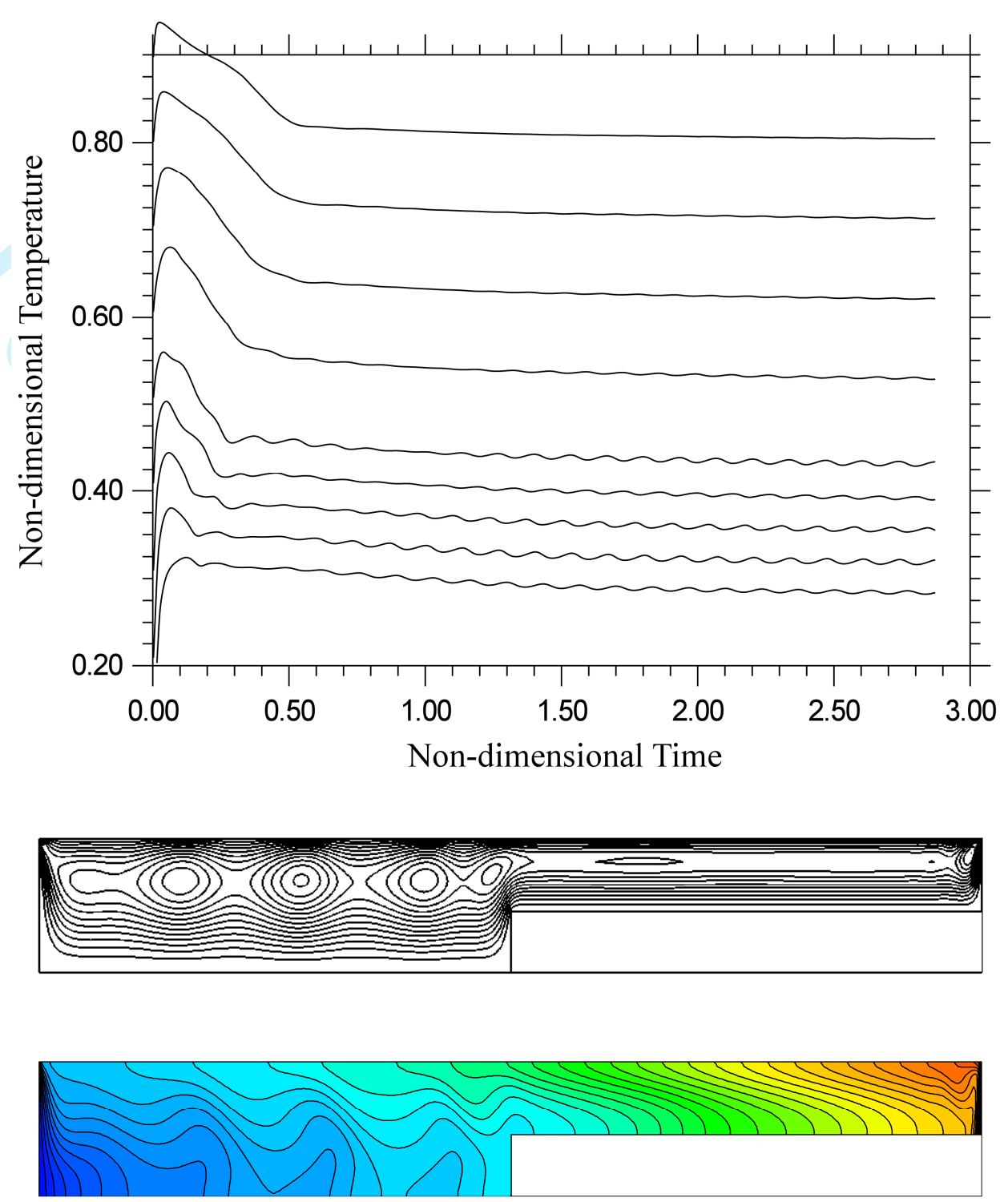

Figure 7: Forward-facing step, $\Lambda=0.45$, adiabatic bottom: a) plot of signals provided by numerical probes, b) snapshot of streamlines $\left(\psi_{\min }=1.35, \psi_{\max }=19\right)$ and $\left.\mathrm{c}\right)$ temperature field at $\mathrm{t}=3$.

\section{$\underline{3.3 \text { Backward-facing step }}$}

Analysis of the results with the backward-facing step leads to the almost immediate (and obvious) conclusion that the conditions established for these cases cannot be regarded as the mirror images of those observed for the forward-facing step. This difference follows obviously from the non-isotropy of the Marangoni flow, that being a shear flow (directed from the hot side to the cold side at the free interface and in the reversed sense in proximity to the bottom), breaks the isotropy of the considered system with respect to the $\mathrm{x}$ coordinate. 
It can be seen that for $\Lambda=0.25$, on average the number of rolls is $\mathrm{N}=10$ and $\mathrm{N}=9$ for adiabatic and conducting conditions on the bottom wall, respectively (Figs. 8 and 9). For these cases, unlike the case of forward-facing step, the amplitude of oscillations is now larger for $x>A / 2$, while their frequency is smaller there.

Again comparison between the two types of thermal conditions on the bottom wall provides some useful information. Indeed, it can be seen that a conducting bottom leads to temperature oscillations with amplitude larger than that obtained for adiabatic conditions, especially where the frequency of the oscillations is higher (it was in the region of smaller frequency for the case of forward-facing step).
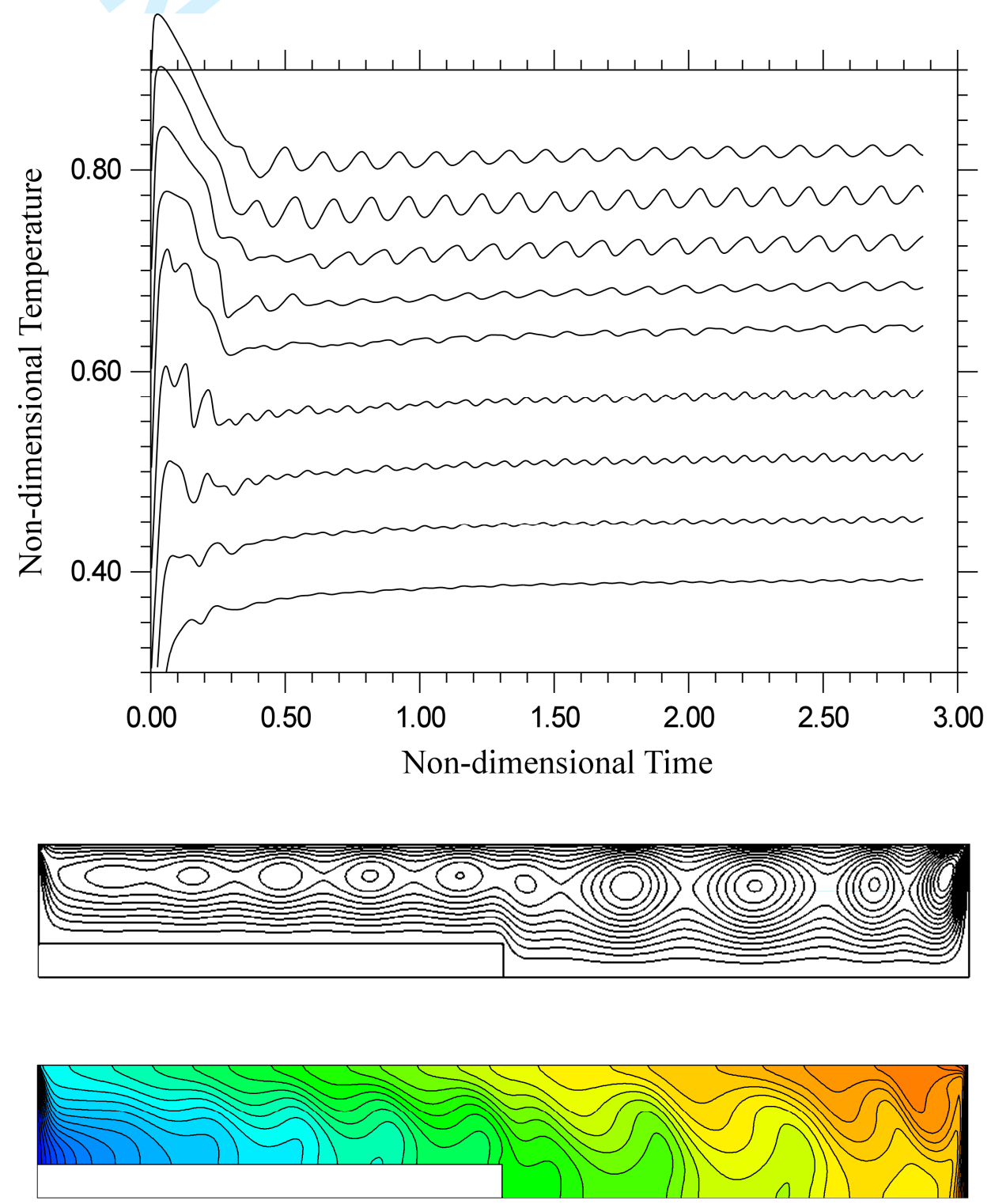

Figure 8: Backward-facing step, $\Lambda=0.25$, adiabatic bottom: a) plot of signals provided by numerical probes, $b$ ) snapshot of streamlines $\left(\psi_{\min }=2, \psi_{\max }=28.8\right)$ and $\left.\mathrm{c}\right)$ temperature field at $\mathrm{t}=3$. 

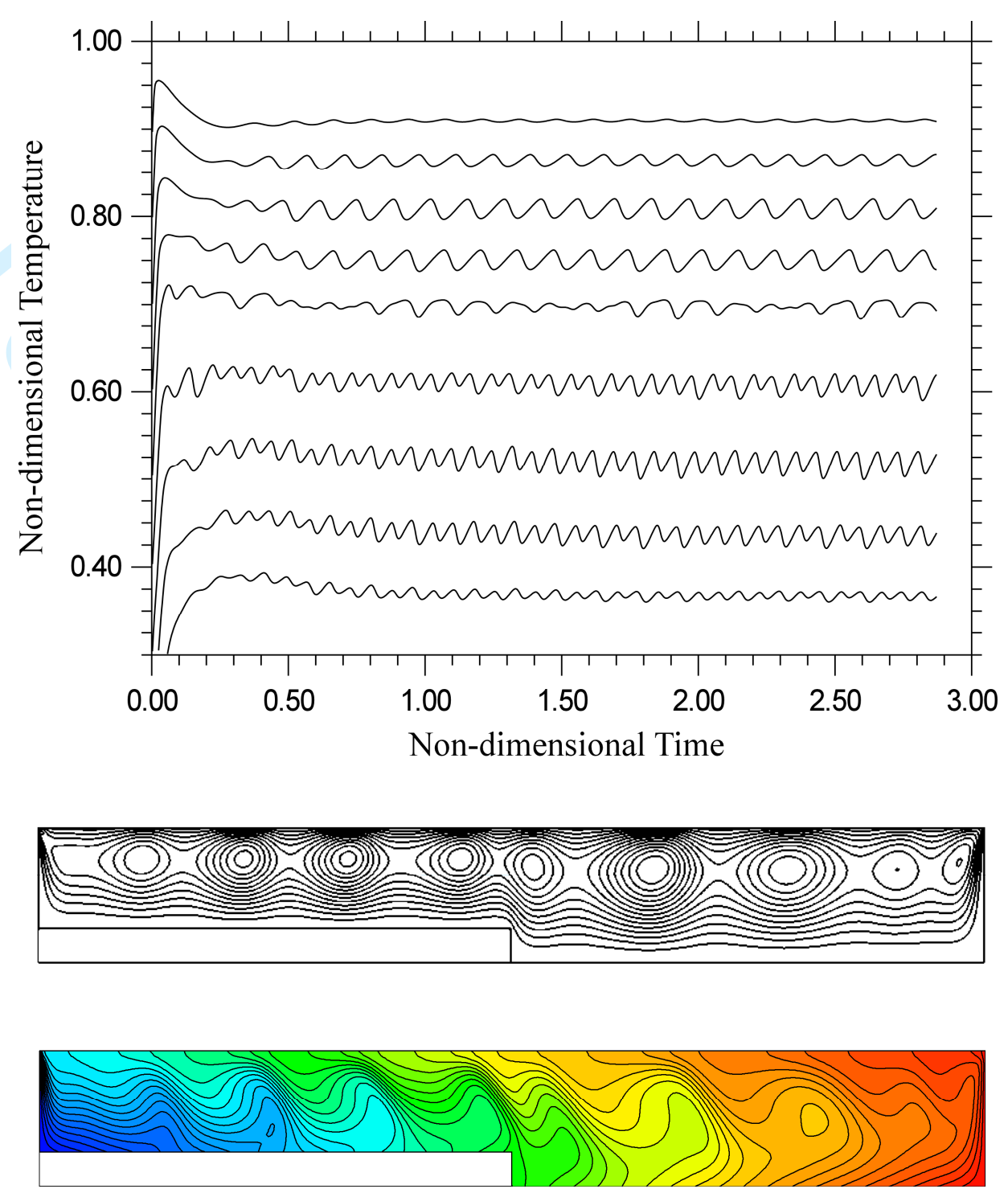

Figure 9: Backward-facing step, $\Lambda=0.25$, conducting bottom: a) plot of signals provided by numerical probes, $b)$ snapshot of streamlines $\left(\psi_{\min }=2.3, \psi_{\max }=31.8\right)$ and $\left.\mathrm{c}\right)$ temperature field at $\mathrm{t}=3$.

An increase in $\Lambda$ (not shown) has an effect similar to that observed for the forward-facing step. For $\Lambda=0.45$ the number of rolls is dramatically reduced ( $N \cong 4$ ), which means the HTW is no longer able to spread over the entire cavity (conditions of parallel flow are established in the region with reduced cross-sectional area).

\section{$\underline{3.4 \text { Obstruction in the center }}$}

Finally we consider the geometry with an obstruction located in the centre (i.e., a reduction of the cross-sectional area for $\mathrm{A} / 4<\mathrm{x}<3 / 4 \mathrm{~A}$ ). 
Some general trends determined from the earlier results seem to be confirmed, i.e. the increase in frequency in the region of reduced cross-sectional are and the related shrinkage of oscillations amplitude (Fig. 10).

The most interesting differences with respect to earlier findings, however, emerge for $\Lambda=0.45$. Indeed, the obstruction located in the cavity center leads to complete stabilization of the flow (no HTW) for the case of adiabatic bottom, while it can survive only in the post-step region $(x>3 / 4$ A) when the conducting wall is considered (Fig. 11).
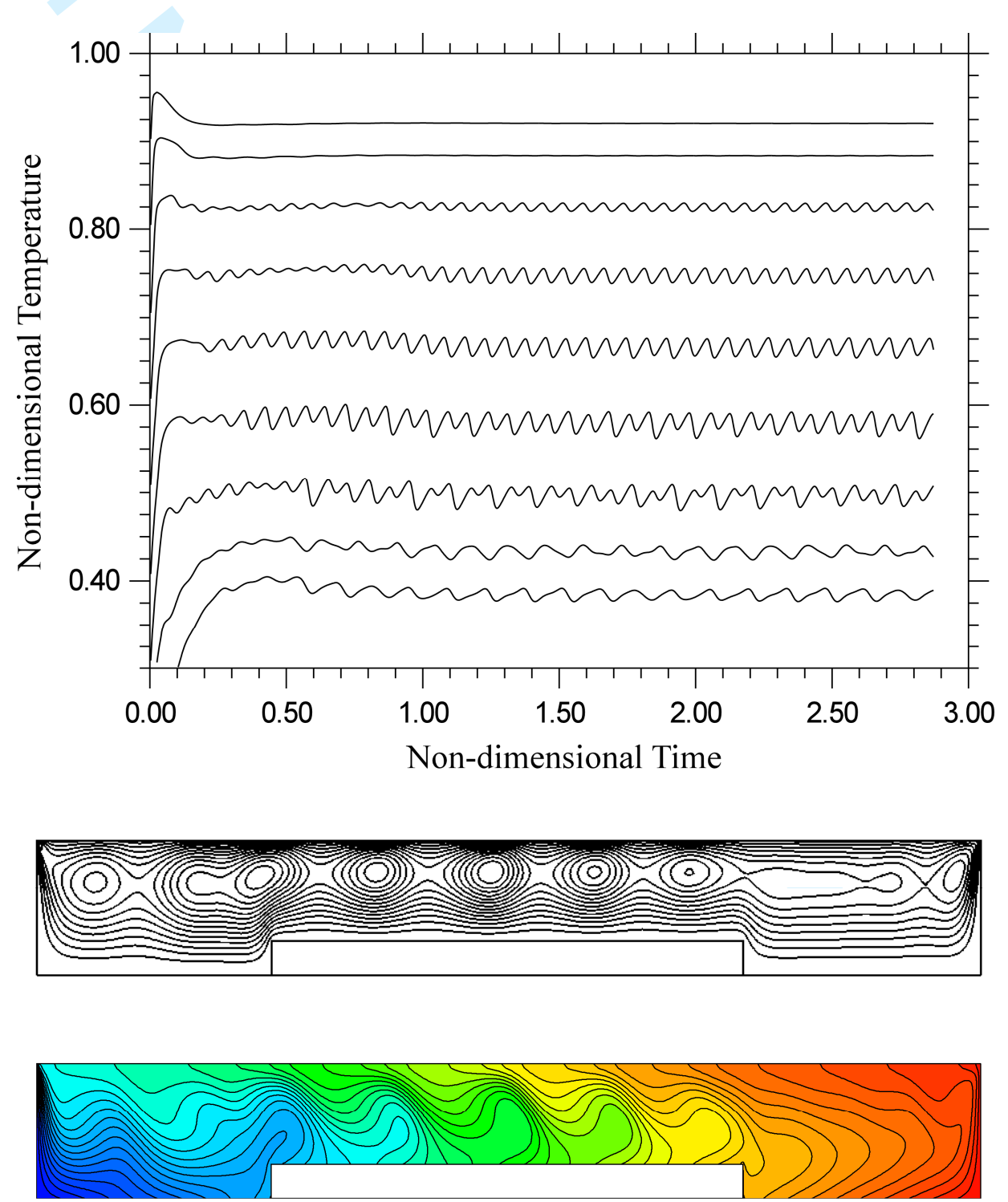

Figure 10: Obstruction, $\Lambda=0.25$, conducting bottom: a) plot of signals provided by numerical probes, b) snapshot of streamlines $\left(\psi_{\min }=1.9, \psi_{\max }=26.8\right)$ and $\left.\mathrm{c}\right)$ temperature field at $\mathrm{t}=3$. 

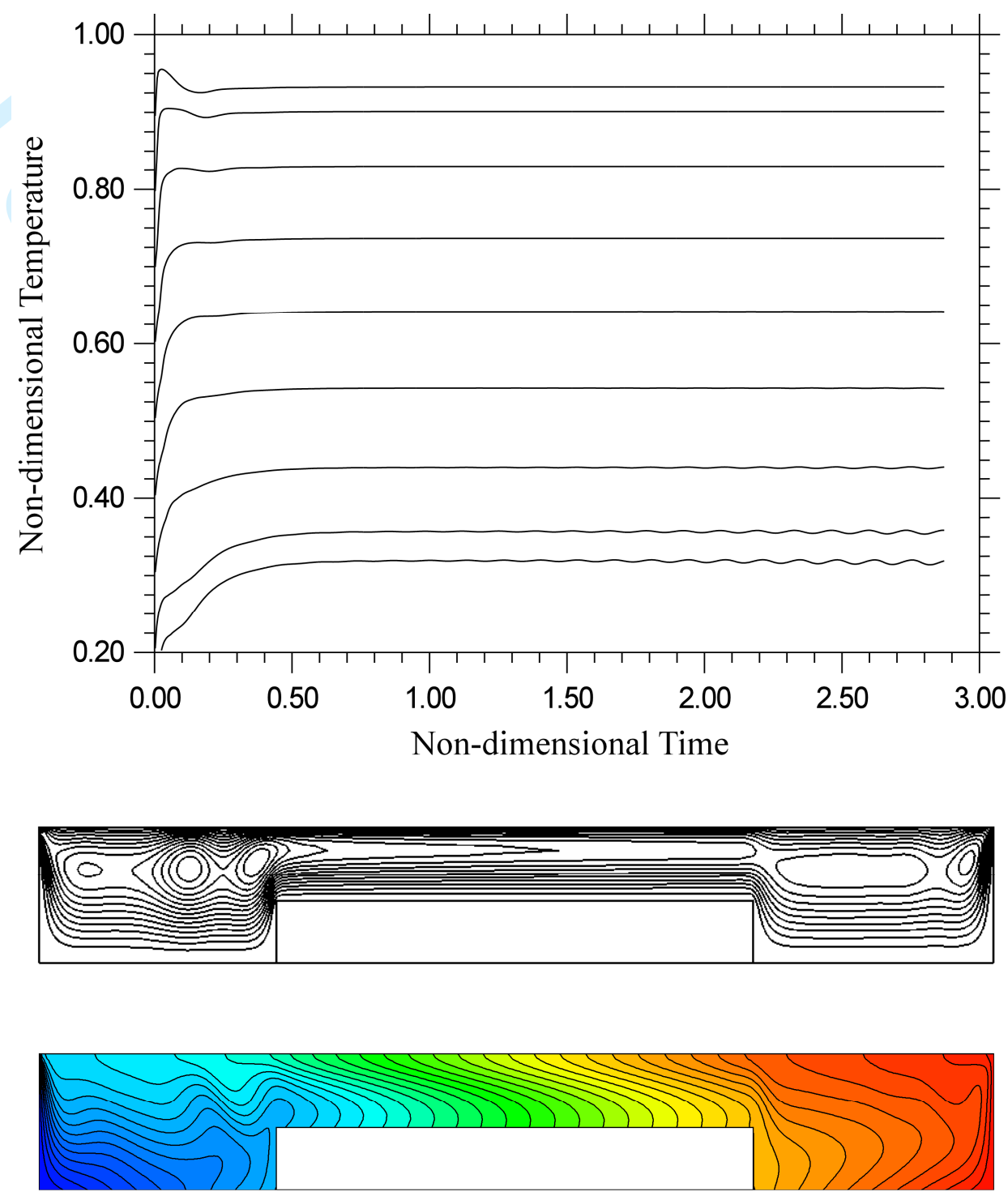

Figure 11: Obstruction, $\Lambda=0.45$, conducting bottom: a) plot of signals provided by numerical probes, b) snapshot of streamlines $\psi_{\min }=1.4, \psi_{\max }=19.5$ ) and c) temperature field at $\mathrm{t}=3$.

A summary of all numerical results (maximum absolute values of the stream function $\psi$ and angular frequency of oscillations measured by the numerical probes $T_{3}$ and $T_{7}$ ) is given in Table $\mathrm{I}$. 
Table I: Summary of numerical results

\begin{tabular}{llllll}
\hline \hline Step & BC & $\Lambda$ & $\psi_{\max }$ & $\omega_{3}$ & $\omega_{7}$ \\
\hline \hline No step & adiabatic & 0 & 19.3 & 45.3 & 45.3 \\
Forward-facing & conducting & 0 & 37.8 & 45.3 & 45.3 \\
& adiabatic & 0.25 & 26.7 & 45.3 & 83.7 \\
& conducting & 0.25 & 40.0 & 45.3 & 81.6 \\
& adiabatic & 0.45 & 20.5 & 45.3 & 0 \\
Backward-facing & conducting & 0.45 & 33.5 & 44.0 & 0 \\
& adiabatic & 0.25 & 30.8 & 84.7 & 45.3 \\
& conducting & 0.25 & 34 & 85.2 & 44.0 \\
& adiabatic & 0.45 & 25.2 & 0 & 44.0 \\
Obstruction & conducting & 0.45 & 29.3 & 0 & 45.3 \\
& adiabatic & 0.25 & 32.1 & 45.3 & 83.7 \\
& conducting & 0.25 & 28.7 & 83.7 & 83.7 \\
& adiabatic & 0.45 & 24.8 & 0 & 0 \\
& conducting & 0.45 & 20.9 & 45.3 & 0 \\
\hline \hline
\end{tabular}

\section{Discussion}

At this stage interesting insights into these phenomena can be derived via critical comparison with the existing literature and related (linear and/or non-linear) studies. Smith and Davis (1983) were the first to elaborate important information about the HTW physical mechanisms: according to their linear stability analysis these waves "derive their energy from the imposed horizontal temperature gradient through horizontal convection when the Prandt number of the liquid is small and from the vertical temperature gradient through vertical convection when it is large". Additional interesting insights into the mechanisms at the basis of these phenomena were also provided by the weakly nonlinear analysis of Smith (1986), who, assuming adiabatic bottom wall and free surface, elucidated the process by which surface temperature disturbances can be amplified or damped according to the Prandtl number and their initial spatial "structure". Some interesting information elaborated on the basis of these studies, which may help to explain the increase in frequency observed here when the cross-sectional area is reduced, is given in the following. 


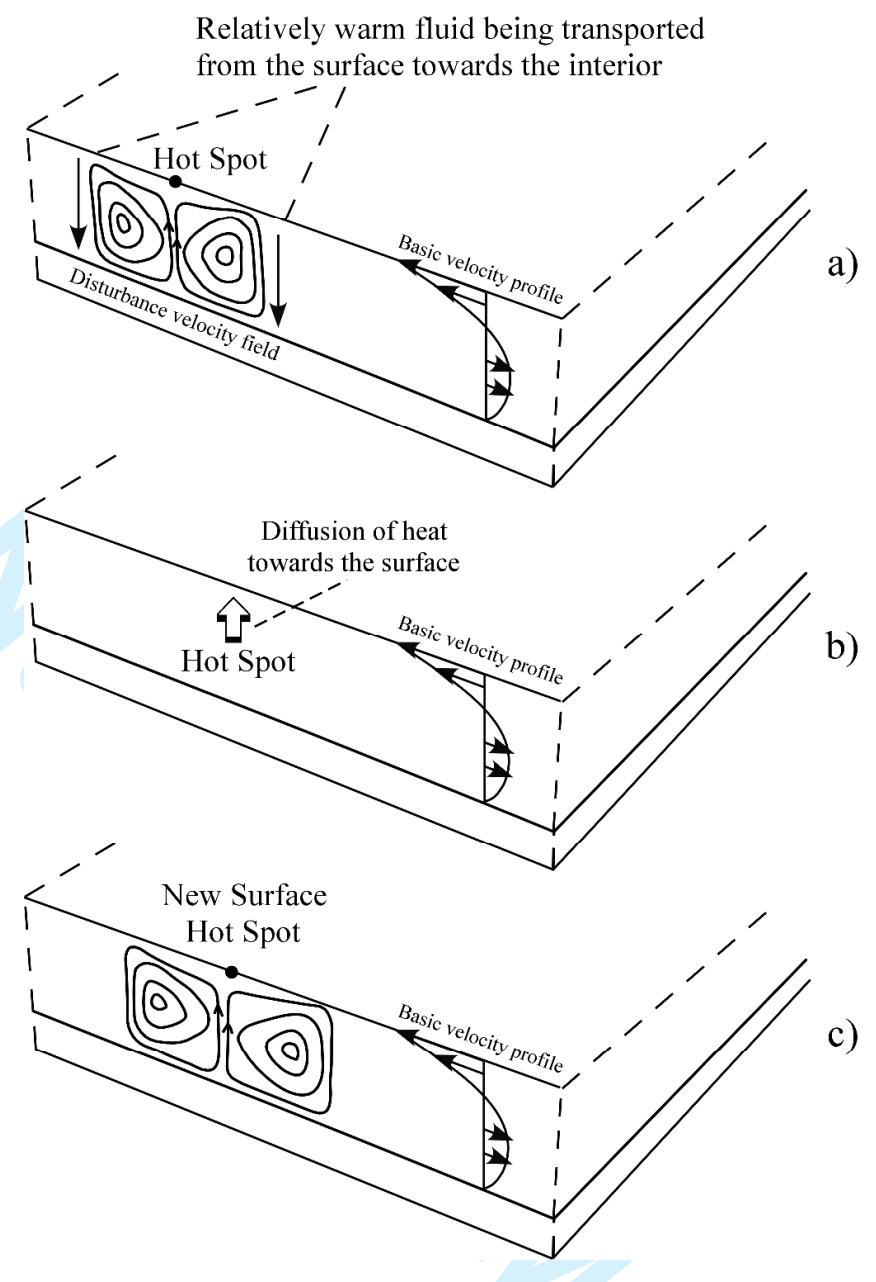

Figure 12: A schematic of the high-Pr mechanism for two-dimensional traveling hydrothermal wave.

Let us start from the simple observation that in the case of large Pr, the flow field in the layer is dominated by viscous effects. Following the arguments provided by Smith (1986), in particular, let us consider a hot disturbance (hot spot) at the free interface located at a generic position $\mathrm{x}$. As shown in Fig. 12a, at the beginning such a spot induces an upward vertical flow. Such upflow can be regarded as the simple natural consequence of the combined effect of thermocapillarity (that brings interfacial fluid from hot regions towards cold ones) and continuity. Clearly, the temperature of the hot spot will decrease as a result of the cooling upflow directly underneath. However, the downflow created by continuity in upstream and downstream positions with respect to the initial hot spots will move surface fluid with a relatively warmer basic-state temperature down to the interior, where the basic-state temperature is colder.

However, hot spots do not form under the surface in both locations where disturbance downflow is present. The temperature perturbations induced by the vertical downflow, in fact, do interact with the basic-state velocity field, which in the interior of the layer is directed from the cold side toward the hot side. For these reasons, as shown in Fig. 12b an interior hot spots forms solely to the right of 
the initial surface hot spot. Such interior hot spot heats the interface of the layer upstream of the original surface spot by diffusion; hence, while the old hot spot decreases in temperature as a result of the cooling upflow, a new hot spot appears upstream of it as a result of diffusive transport of heat in the vertical direction.

This new spot produces the same recirculating velocity field as the old one; thereby, the process depicted above can start again with initial location slightly shifted upstream with respect to the earlier one (Fig. 12c). It is evident that the key to this mechanism is the interior hot spot that drives the instability by conductively heating the interface (the energy for the spot is clearly extracted, as outlined before, from the vertical basic-state temperature field by vertical convection).

With such arguments in mind, the strong increase in frequency found in the regions where the cross-sectional area is reduced (see Table I) can be explained simply taking into account that by shortening the distance between the surface and the return flow, the presence of an obstruction will naturally lead to an acceleration of the overall process depicted above. Indeed, while the driving force does not change (the surface Marangoni stress depends essentially on the properties of the considered fluid and the applied temperature gradient), the time required for the diffusive transport of heat in the vertical direction will be significantly reduced.

\section{Conclusions}

The influence of several types of boundary conditions, both of kinematic and thermal nature (relative position of step and flow direction, expansion/compression ratio, thermal boundary condition at the bottom wall) on the preferred mode of instability of Marangoni flow in a liquid layer of finite extent has been examined under the constraint of two-dimensional flow. Though our model cannot account for the spanwise component of the emerging travelling disturbance, it has been shown that sudden changes in the geometry of the container can induce significant modification of the wave component travelling in the upstream direction. The presence of an obstacle has, in general, a two-fold effect on the property of such a disturbance: it leads to an increase in the velocity of propagation (as witnessed by the corresponding increase in the temperature signal frequency) and determines a shrinkage of the corresponding amplitude. This, in turn, is reflected in observable remarkable changes of the convective rolls structure and related spatio-temporal behaviour. The rolls associated with the hydrothermal wave, which spread continuously from the cold side towards the hot side, are slowed down or accelerated according to whether they are located in regions of smaller or larger cross-sectional area, respectively. Most notably, complete suppression of the oscillatory behaviour is possible in the regions of reduced cross-sectional area when the thickness of the obstacle or step is sufficiently large.

Though some general trends can be identified, the dependence of the phenomena on the geometrical parameters, however, is not trivial due to the intrinsic nature of the Marangoni flow, that being a shear flow (directed from the hot side to the cold side at the free interface and in the reversed sense 
in proximity to the bottom), breaks the isotropy of the considered system with respect to the $\mathrm{x}$ coordinate.

The type of thermal boundary condition at the bottom wall (adiabatic or conducting) has also a nonnegligible impact on the emerging dynamics, as witnessed by the increased ability of the hydrothermal wave to resist the stabilizing (damping) effect exerted on it by the reduction of the available cross-sectional area when a conducting bottom wall is considered.

With the due limitations, this work has demonstrated the capability of a relatively simple (existing) computational framework to capture some still poorly known aspects being related to the typical instabilities of Marangoni flows in layers and rectangular cavities having a non-ordinary geometry. The present analysis has been carried out under the optimistic hope that it will act as a platform onto which we can build new models able to describe similar dynamics in more complex (3D) configurations, closely resembling effective industrial crucibles.

\section{References}

Abu-Mulaweh H.I., (2002), Effects of backward- and forward-facing steps on turbulent natural convection flow along a vertical flat plate, International Journal of Thermal Sciences, 41(4): $376-385$.

Abu-Mulaweh H.I., (2003), A review of research on laminar mixed convection flow over backward- and forward-facing steps, International Journal of Thermal Sciences, 42(9): 897909.

Abu-Mulaweh H.I., (2005), Turbulent mixed convection flow over a forward-facing step - the effect of step heights, International Journal of Thermal Sciences, 44(2): 155-162.

Bucchignani E., (2004), Numerical characterization of hydrothermal waves in a laterally heated shallow layer, Phys Fluids, 16(11): 3839-3849.

Burguete J., Mukolobwiez N., Daviaud N., Garnier N., Chiffaudel A., (2001), Buoyantthermocapillary instabilities in extended liquid layers subjected to a horizontal temperature gradient, Phys. Fluids, 13 (10): 2773-2787

Chen G., Moiola J.L. and Wang H.O., (2000), Bifurcation Control: Theories, Methods and Applications, International Journal of Bifurcation and Chaos, 10(3): 511-548

Chorin A.J., (1968), Numerical solutions of the Navier-Stokes equations, Math. Comput., 22: 745762.

Daviaud F. and Vince J.M., (1993), Traveling waves in a fluid layer subjected to a horizontal temperature gradient, Phys. Rev. E, 48: 4432-4436.

De Saedeleer C., Garcimartin A., Chavepeyer G., Platten J.K., Lebon G., (1996), The instability of a liquid layer heated from the side when the upper surface is open to air, Phys. Fluids, 8(3): 670-676. 
Dihmani N., Amraqui S., Mezrhab A. and Laraqi N., (2012), Numerical Modelling of Rib Width and Surface Radiation Effect on Natural Convection in a Vertical Vented and Divided Channel, Fluid Dyn. Mater. Process., 8(3): 311-322.

Garcimartìn A., Mukolobwiez N., and Daviaud F., (1997), Origin of surface waves in surface tension driven convection, Phys. Rev. E 56: 1699-1705.

Gillon P. and Homsy G.M., (1996), Combined thermocapillary-buoyancy convection in a cavity: an experimental study, Phys. Fluids, 8:2953-2963.

Gresho P.M., (1991), Incompressible fluid dynamics: some fundamental formulation issues, Ann. Rev Fluid Mech., 23: 413-453.

Gresho P. M. and Sani R. T. (1987), On pressure boundary conditions for the incompressible Navier-Stokes equations, Int. J. Num. Methods Fluids, 7: 1111-1145.

Guermond J.-L. and Quartapelle L., (1998), On stability and convergence of projection methods based on pressure Poisson equation, Int. J. Numer. Meth. Fluids, 26: 1039-1053.

Guermond J.-L., Minev P. and Shen J., (2006), An Overview of Projection Methods for Incompressible Flows. Comput. Methods, Comput. Methods Appl. Mech. Eng., 195: 60116045.

Harlow F.H. and Welch J.E. (1965), Numerical calculation of time-dependent viscous incompressible flow with free surface, Phys. Fluids, 8: 2182-2189.

Ladyzhenskaya O.A., (1969), The Mathematical Theory of Viscous Incompressible Flow, Gordon and Breach, 2nd Edition, New York - London, 1969.

Lappa M. and Savino R., (1999), Parallel solution of the three-dimensional Marangoni flow instabilities in liquid bridges, Int. J. Num. Meth. Fluids, 31: 911-925.

Lappa M., (2005), Thermal convection and related instabilities in models of crystal growth from the melt on earth and in microgravity: Past history and current status, Cryst. Res. Technol., 40(6): 531-549.

Lappa M., (2007a), Secondary and oscillatory gravitational instabilities in canonical threedimensional models of crystal growth from the melt, Part2: Lateral heating and the Hadley circulation, Comptes Rendus Mécanique, 335(5-6): 261-268.

Lappa M., (2007b), Secondary and oscillatory gravitational instabilities in canonical threedimensional models of crystal growth from the melt, Part1: Rayleigh-Bènard systems, Comptes Rendus Mécanique, 335(5-6): 253-260.

Lappa M., (2013), Assessment of the role of axial vorticity in the formation of Particle Accumulation Structures (PAS) in supercritical Marangoni and hybrid thermocapillaryrotation-driven flows, Phys. Fluids, 25(1) 012101 (11 pages).

Lappa M., (2014), Stationary Solid Particle Attractors in Standing Waves, Phys. Fluids, 26(1), 013305 (12 pages).

Lappa M., (2016), On the onset of multi-wave patterns in laterally heated floating zones for slightly supercritical conditions, Physics of Fluids, 28(12): 124105 (22 pages) 
Lappa M., Fluids, Materials and Microgravity: Numerical Techniques and Insights into the Physics (Elsevier Science, Oxford, England, 2004).

Lappa M., Rotating Thermal Flows in Natural and Industrial Processes (John Wiley \& Sons, Chichester, England, 2012).

Lappa M., Savino R. and Monti R., (2000), Influence of buoyancy forces on Marangoni flow instabilities in liquid bridges, Int. J. Num. Meth. Heat Fluid Flow, 10 (7): 721-749.

Lappa M., Thermal Convection: Patterns, Evolution and Stability (John Wiley \& Sons, Chichester, England, 2009).

Lappa M., Yasushiro S., Imaishi N., (2003) 3D numerical simulation of on ground Marangoni flow instabilities in liquid bridges of low Prandtl number fluid, Int. J. Num. Meth. Heat Fluid Flow, 13 (3): 309-340.

Li Y.R., Peng L., Akiyama Y., Imaishi N., (2003), Three-dimensional numerical simulation of thermocapillary flow of moderate Prandtl number fluid in an annular pool, J. Cryst. Growth, 259: 374-387.

Li Y.R., Peng L., Shi W.Y. and Imaishi N., (2006), Convective instabilities in annular pools, Fluid Dyn. Mater. Process., 2(3): 153-166.

Mahrouche O., Najam M., El Alami M., Faraji M., (2013), Mixed Convection Investigation in an Opened Partitioned Heated Cavity, Fluid Dyn. Mater. Process., 9(3): 235-250.

Melnikov D.E., Shevtsova V.M. and Legros J.C., (2005), Route to aperiodicity followed by high Prandtl-number liquid bridge. 1-g case, Acta Astronautica, 56(6): 601-611.

Melnikov D.E., Shevtsova V.M. and Legros, J.C., (2004), Onset of temporal aperiodicity in high Prandtl number liquid bridge under terrestrial conditions, Phys. Fluids, 16(5): 1746-1757.

Meskini A., Najam M. and El Alami M., (2011), Convective Mixed Heat Transfer in a Square Cavity with Heated Rectangular Blocks and Submitted to a Vertical Forced Flow, Fluid Dyn. Mater. Process., 7(1): 97-110.

Ohta H. and Hosono H., (2004), Transparent oxide optoelectronics, Materials Today, 7(6): 42-51. Pelacho M.A. and Burguete J., (1999), Temperature oscillations of hydrothermal waves in thermocapillary-buoyancy convection, Phys. Rev. E 59: 835-840.

Pelacho M.A., Garcimartın A., and Burguete J., (2000), Local Marangoni number at the onset of hydrothermal waves, Phys. Rev. E, 62: 477-483.

Pelacho M.A., Garcimartin A., Burguete J., (2001), Travel Instabilities in Lateral Heating, Int. J. Bifurcat. Chaos, 11(11): 2881-2886.

Peltier L. and Biringen S., (1993), Time-dependent thermocapillary convection in a rectangular cavity: numerical results for a moderate Prandtl number fluid, J. Fluid Mech., 257: 339-357.

Priede J. and Gerbeth G., (1997), "Influence of thermal boundary conditions on the stability of thermocapillary-driven convection at low Prandtl numbers", Phys. Fluids, 9: 1621-1634.

Quartapelle L., (1993), Numerical Solution of the Incompressible Navier-Stokes Equations, International Series of Numerical Mathematics vol. 113, Birkäuser, 1993. 
Riley R.J. and Neitzel G.P., (1998), Instability of thermocapillary-buoyancy convection in shallow layers. Part 1. Characterization of steady and oscillatory instabilities, J. Fluid Mech., 359: 143-164.

Rouijaa H., El Alami M., El Alami Semma and Najam M., (2011), Natural Convection in an Inclined T-Shaped Cavity, Fluid Dyn. Mater. Process., 7(1): 57-70.

Schwabe D., Moller U., Schneider J. and Scharmann A., (1992), Instabilities of shallow dynamic thermocapillary liquid layers, Phys. Fluids A, 4, 2368-2381.

Schwabe D., Zebib A and Sim B.C., (2003), Oscillatory thermocapillary convection in open cylindrical annuli. Part 1. Experiments under microgravity, J. Fluid Mech., 491: 239-258.

Shevtsova V., Mialdun A., Kawamura H., Ueno I., Nishino K., Lappa M. (2014), The JEREMIProject on Thermocapillary Convection in Liquid Bridges. Part B: Impact of Co-axial Gas Flow, Fluid Dyn. Mater. Process., 10(2), 197-240.

Shevtsova V., et al., (2011), Onset of Hydrothermal Instability in Liquid Bridge. Experimental Benchmark, Fluid Dyn. Mater. Process, 7(1): 1-28.

Shevtsova V., Melnikov D.E., Nepomnyashchy A., (2009), New flow regimes generated by mode coupling in buoyant-thermocapillary convection, Phys. Rev. Lett., 102: 134503.

Shevtsova V.M., Melnikov D.E. and Legros J.C., (2003), Multistability of oscillatory thermocapillary convection in a liquid bridge, Phys. Rev. E, 68 (6): 066311 (14 pages).

Shevtsova V.M., Melnikov D.E., Legros J.C., (2001), Three-dimensional simulations of hydrodynamical instability in liquid bridges: influence of temperature-dependent viscosity, Phys Fluids, 13: 2851-2865.

Shi W. Y. and Imaishi N., (2006), Hydrothermal waves in differentially heated shallow annular pools of silicone oil, J. Cryst. Growth, 290: 280-291.

Smith M.K, (1986), Instability mechanism in dynamic thermocapillary liquid layers, Phys. Fluids, 29 (10): 3182-3186.

Smith M.K. and Davis S.H. (1983), Instabilities of dynamic thermocapillary liquid layers. Part 1: convective instabilities, J. Fluid Mech 132: 119-144.

Tang Z.M. and Hu W.R., (2005), Hydrothermal Wave in a Shallow Liquid Layer, Microgravity Sci. Tech., 16(1): 253-258.

Temam R., (1968), Une méthode d'approximation de la solution des équations de Navier-Stokes, Bull. Soc. Math. France, 98: 115-152.

Ueno I., Tanaka S. and Kawamura H., (2003), Oscillatory and chaotic thermocapillary convection in a half-zone liquid bridge, Phys. Fluids, 15(2): 408-416.

Xu J. and Zebib A., (1998), Oscillatory two- and three-dimensional thermocapillary convection, $J$. Fluid Mech., 364: 187-209. 